\title{
Improving biofeedback for the treatment of fecal incontinence in women: implementation of a standardized multi-site manometric biofeedback protocol
}
A. D. Markland ${ }^{1,2}$
J. E. Jelovsek ${ }^{3}$
W. E. Whitehead ${ }^{4,5}$
D. K. Newman ${ }^{6}$ ।
U. U. Andy ${ }^{7}$
K. Dyer ${ }^{8}$
I. Harm-Ernandes ${ }^{9}$
S. Cichowski ${ }^{10}$
J. McCormick ${ }^{1,2}$ ।
C. $\operatorname{Rardin}^{11}$
G. Sutkin ${ }^{12}$
A. Shaffer ${ }^{13}$
S. Meikle ${ }^{14}$
on behalf of the Pelvic Floor
Disorders Network

\footnotetext{
${ }^{1}$ Division of Gerontology, Geriatrics and Palliative Care, Department of Medicine, University of Alabama at Birmingham, Birmingham, AL, USA

${ }^{2}$ Geriatric Research, Education, and Clinical Center, The Birmingham Veterans Affairs Medical Center, Birmingham, AL, USA

${ }^{3}$ Obstetrics, Gynecology \& Women's Health Institute, Cleveland Clinic, Cleveland, OH, USA

${ }^{4}$ Division of Gastroenterology and Hepatology, Department of Medicine, University of North Carolina at Chapel Hill, Chapel Hill, NC, USA

${ }^{5}$ Division of Urogynecology, Department of Obstetrics and Gynecology, University of North Carolina at Chapel Hill, Chapel Hill, NC, USA

${ }^{6}$ Division of Urology, Department of Surgery, University of Pennsylvania Perelman School of Medicine, Philadelphia, PA, USA

${ }^{7}$ Department of Obstetrics and Gynecology, University of Pennsylvania Perelman School of Medicine, Philadelphia, PA, USA

${ }^{8}$ Department of Obstetrics and Gynecology, Kaiser Permanente, San Diego, CA, USA

${ }^{9}$ Department of Obstetrics and Gynecology, Duke University Medical Center, Durham, NC, USA

${ }^{10}$ Departments of Obstetrics and Gynecology and Surgery, University of New Mexico Health Sciences Center, Albuquerque, NM, USA

${ }^{11}$ Department of Obstetrics and Gynecology, Alpert Medical School of Brown University, Providence, RI, USA

${ }^{12}$ Department of Obstetrics, Gynecology and Reproductive Sciences, University of Pittsburgh Medical Center, Pittsburgh, PA, USA

${ }^{13}$ RTI International, Research Triangle Park, NC, USA

${ }^{14}$ The Eunice Kennedy Shriver National Institute of Child Health and Human Development, National Institutes of Health, Bethesda, MD, USA
}

\section{Correspondence}

Alayne D. Markland, DO, MSc, Division of Gerontology, Geriatrics and Palliative Care, Department of Medicine, University of Alabama at Birmingham, $70019^{\text {th }}$ Street South, Birmingham, AL 35294, USA.

Email: amarkland@uabmc.edu

View the podcast on this paper at the following sites: iTunes: https://itunes.apple.com/gb/podcast/ neurogastroenterology-motility/id1183308675 YouTube: https://youtu.be/OgRay1WjiNs

\begin{abstract}
Background: Standardized training and clinical protocols using biofeedback for the treatment of fecal incontinence (FI) are important for clinical care. Our primary aims were to develop, implement, and evaluate adherence to a standardized protocol for manometric biofeedback to treat FI.

Methods: In a Pelvic Floor Disorders Network (PFDN) trial, participants were enrolled from eight PFDN clinical centers across the United States. A team of clinical and equipment experts developed biofeedback software on a novel tablet computer platform for conducting standardized anorectal manometry with separate manometric biofeedback protocols for improving anorectal muscle strength, sensation, and urge resistance. The training protocol also included education on bowel function, anal sphincter exercises, and bowel diary monitoring. Study interventionists completed online training prior to attending a centralized, standardized certification course. For the certification, expert trainers assessed the ability of the interventionists to perform the protocol components for a paid volunteer who acted as a standardized patient. Postcertification, the trainers audited interventionists during trial implementation to improve protocol adherence.
\end{abstract}


Key Results: Twenty-four interventionists attended the in-person training and certification, including 46\% advanced practice registered nurses (11/24), 50\% (12/24) physical therapists, and $4 \%$ physician assistants (1/24). Trainers performed audio audits for $88 \%(21 / 24)$, representing 84 audited visits. All certified interventionists met or exceeded the prespecified $80 \%$ pass rate for the audit process, with an average passing rate of $93 \%$.

Conclusions \& Inferences: A biofeedback protocol can be successfully imparted to experienced pelvic floor health care providers from various disciplines. Our process promoted high adherence to a standard protocol and is applicable to many clinical settings.

\section{KEYWORDS}

anal sphincter exercises, anorectal manometry, factorial design, fecal incontinence, manometryassisted biofeedback, pelvic floor disorders, randomized placebo-controlled trial, treatment fidelity, urge resistance training

\section{1 | BACKGROUND}

Fecal incontinence (FI) is the unintentional loss of solid or liquid stool. Fecal incontinence has a reported prevalence ranging from $7 \%$ to $15 \%$ in community-dwelling women; however, these numbers may underestimate the actual prevalence as women often fail to discuss, and providers neglect to ask about, this embarrassing condition. ${ }^{1}$ Women with FI limit time away from home and avoid social situations, which results in a loss of self-respect and confidence. ${ }^{2,3}$

The three most common contributing causes of $\mathrm{Fl}$ are (i) weakness of the pelvic floor muscles due to structural or neurological injuries, (ii) deficits in the ability to perceive rectal filling, sometimes referred to as rectal hyposensitivity, and (iii) inability to delay defecation sometimes referred to as rectal hypersensitivity or urge FI. ${ }^{4,5}$ Diarrhea is also a well-established risk factor which may interact with these three physiological deficits to precipitate or exacerbate $\mathrm{FI}^{1,4}$

The first-line therapeutic options for FI typically include behavioral therapy, medications and dietary changes. ${ }^{4,5}$ The American College of Gastroenterology and the American Gastroenterological Association both recommend biofeedback for the treatment of $\mathrm{Fl}^{6}{ }^{6}$ However, a conference held at the National Institutes of Health entitled "Developing a Clinical Research Agenda for Fecal Incontinence" found that randomized controlled trials of biofeedback have yielded inconsistent results and concluded that "research is needed to standardize the treatment protocols and the training of biofeedback therapists." ${ }^{4}$ Biofeedback treatment protocols for $\mathrm{Fl}$ include efforts to strengthen pelvic floor muscles, and many also include techniques for increasing the ability to sense rectal filling. ${ }^{7-11}$ However, the details of when and how sensory training should be combined with strength training have not been examined, and only one recent study evaluated the effectiveness of sensory and urge resistance training. ${ }^{8}$ The purpose of this article was to describe a standardized biofeedback with anal exercises treatment protocol that was developed and validated for a specific multicenter clinical trial. ${ }^{12}$ We will refer to this protocol as the "biofeedback protocol" hence forward.

\section{Key Points}

- Separate biofeedback protocols addressed the three most common causes for fecal incontinence: sphincter weakness, rectal hyposensitivity, and urge fecal incontinence (hypersensitivity).

- Centralized training along with the use of standardized patient models was an effective method for teaching biofeedback protocols.

- Auditing of the biofeedback visits revealed high adherence to the biofeedback protocols.

The aims of this article were: (i) to describe the rationale and theoretical background for the biofeedback protocol, (ii) to provide a general description of the biofeedback training and biofeedback protocol supplemented with access to online curriculum and training resources, (iii) to describe evaluation efforts for biofeedback protocol adherence, and (iv) to describe monitoring the fidelity of the interventionists to the biofeedback protocol over time in the clinical trial, Controlling Anal Incontinence by Performing Anal Exercises With Biofeedback or Loperamide (CAPABLe) Study (NCT02008565).

\section{2 | METHODOLOGY}

\section{1 | Study overview}

The protocol and study methods for the CAPABLe study are published elsewhere. ${ }^{12}$ Eligible participants are randomized to one of four groups in a two-by-two factorial design: (i) usual care (educational pamphlet) with oral placebo, (ii) oral loperamide at a minimum dose of $2 \mathrm{mg}$ taken orally every other day to a maximum of $8 \mathrm{mg}$ daily with usual care (educational pamphlet), (iii) anal sphincter exercise training with anorectal manometry (ARM)-assisted biofeedback 
TABLE 1 Biofeedback protocol components and actions based on study measures

\begin{tabular}{|c|c|c|c|}
\hline Biofeedback protocol & Bowel diary & ARM finding & Action for interventionist \\
\hline \multirow{2}{*}{ Strength training } & \multirow{2}{*}{$\begin{array}{l}\text { Bowel accidents occur } \\
\text { with or without urge }\end{array}$} & $\begin{array}{l}\text { Squeeze duration at } 50 \% \text { of } \\
\text { maximal squeeze pressure for } \\
<10 \text { seconds }\end{array}$ & $\begin{array}{l}\text { Encourage pelvic floor muscle exercises at home. } \\
\text { Set realistic weekly goals to reach a } 10 \text {-second } \\
\text { squeeze with equal periods of muscle rest at above } \\
50 \% \text { of the maximal contraction strength by the end } \\
\text { of the study visits. } \\
\text { Employ strength training biofeedback protocol. }\end{array}$ \\
\hline & & $\begin{array}{l}\text { Rectal pressure increases } \\
>10 \mathrm{mmHg} \text { when squeezing }\end{array}$ & $\begin{array}{l}\text { Identify this as something that increases the risk of } \\
\text { FI. } \\
\text { Employ strength training biofeedback protocol but } \\
\text { focus on isolating the sphincter muscles by keeping } \\
\text { rectal pressure below } 10 \mathrm{mmHg} \text { during squeezes. }\end{array}$ \\
\hline Urge resistance training & $\begin{array}{l}\text { One or more bowel } \\
\text { accidents that are } \\
\text { preceded by a strong } \\
\text { sense of urgency }\end{array}$ & $\begin{array}{l}\text { Abnormally low maximum } \\
\text { tolerable volume on ARM } \\
(<100 \mathrm{~mL})\end{array}$ & $\begin{array}{l}\text { Provide positive guidance that rectal capacity can be } \\
\text { improved in future study visits by employing urge } \\
\text { resistance training biofeedback protocol. }\end{array}$ \\
\hline
\end{tabular}

with usual care (educational pamphlet) plus oral placebo, and (iv) combination oral loperamide with anal sphincter exercise training with manometry-assisted biofeedback with usual care (educational pamphlet). The study population consists of adult women with at least monthly $\mathrm{Fl}$ over the last 3 months that is bothersome enough to seek and desire treatment. Women with predominant extremes of stool consistency on the Bristol Stool Form are excluded as patients with constipation are not candidates for potentially constipating agents such as loperamide and patients with chronic watery diarrhea may have a variety of causes for their diarrhea that need to be treated such as infectious etiologies. ${ }^{12}$ The purpose of this article was to describe the biofeedback training and biofeedback protocol in sufficient detail that it can be used by other investigators who utilize biofeedback for Fl. All biofeedback study measures, tasks, and the biofeedback protocol manual of procedures are in the Data S1.

\section{2 | Principles of the biofeedback protocols}

Because the behavioral training techniques for the three physiological deficits (sphincter muscle weakness, hyposensitivity, and hypersensitivity) differ, we developed separate clinical decision algorithms and accompanying training protocols for each (Table 1). We also collaborated with an equipment manufacturer to develop customized software which would guide interventionists through each protocol using on-screen prompts (Fig. 1) and provide a summary for each training session showing the best response achieved during each biofeedback protocol to facilitate comparisons across sessions. The session report shows the "best" response rather than the average response because performance is expected to improve during biofeedback training.

\section{3 | Biofeedback protocols: components}

Biofeedback protocol treatments occur over six 1- to 2-hour sessions spaced approximately 2 weeks apart to allow the participant time to practice new skills and to build strength through daily practice, results of which are captured on a bowel accident and symptom diary (bowel diary). Although there is some individualization of training sessions

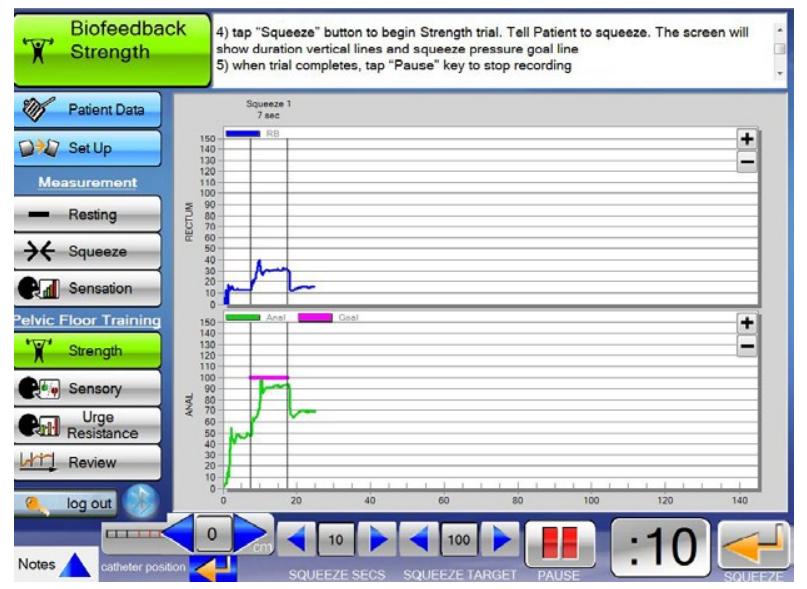

FIGURE 1 Biofeedback software with on-screen protocol prompts from a tablet computer device*.

*http://medspira.com/products/mcompass/anorectal-manometry/ 
TABLE 2 Overall goals of the biofeedback protocol

\begin{tabular}{|c|c|}
\hline Goal of training & Biofeedback goal \\
\hline Increase contraction strength at sphincter & Contraction strength of $100 \mathrm{mmHg}$ or higher \\
\hline Increase contraction duration at sphincter & $\begin{array}{l}\text { Maintain contraction at } 50 \% \text { of the maximal contraction pressure for at } \\
\text { least } 10 \text { seconds with equal periods of relaxation }\end{array}$ \\
\hline $\begin{array}{l}\text { Maintain constant rectal balloon pressure while increasing sphincter } \\
\text { strength/duration }\end{array}$ & Rectal balloon pressure does NOT increase $>10 \mathrm{mmHg}$ with squeeze \\
\hline Increase sensation of rectal balloon filling (improve sensory threshold) & Sensation for $15 \mathrm{~mL}$ volume of air injected into the rectal balloon \\
\hline Increase strong urge sensation threshold & Volume should reach $100 \mathrm{~mL}$ or more \\
\hline $\begin{array}{l}\text { Generalization techniques (appropriate for the sensory and urge } \\
\text { resistance training biofeedback groups) }\end{array}$ & $\begin{array}{l}\text { Using the techniques ("hypervigilance," "stop, don't run," and "delay } \\
\text { defecation") on a regular/daily basis outside of the clinical setting to } \\
\text { avoid accidental bowel leakage }\end{array}$ \\
\hline
\end{tabular}

based on differences among participants in physiological deficits and rates of change, each visit includes the following components (see Table S1 for details).

1. Review the daily bowel diary with participant and provide motivational feedback to continue home exercise plan.

2. Perform ARM examination. The ARM findings should be compared to previous sessions and motivational feedback should be provided. The ARM data, in combination with the bowel diary, guide the interventionist on whether to employ sensory training or urge resistance training; see Table 1.

3. Perform manometric biofeedback protocols using on-screen prompts for instructions. All participants receive strength training. Sensory training and/or urge resistance training is added based on the ARM findings and bowel diary (Table 1):

a. Strength training to improve maximum squeeze pressure, squeeze duration, and ability to isolate (i.e., to squeeze pelvic floor muscles without simultaneously increasing rectal pressure).

b. Sensory training (only if there is evidence of hyposensitivity) to decrease the threshold for perception of rectal balloon distention.

c. Urge resistance training (only if there is evidence of hypersensitivity) to increase the participant's tolerance for larger rectal balloon distensions without experiencing an intolerably strong urge to defecate.

4. At the conclusion of the session, instructions for home practice are given and include an exercise prescription, a daily exercise record for the participant to record exercises, a 7-day bowel diary, and a behavioral instructional handout for generalization at home. An interventionist log is used to record the key components of the bowel diary, ARM values, biofeedback goals, and exercise record activity. This facilitates providing feedback to the patient on their progress.

Important concepts underlying biofeedback training that were used to develop the protocol are shaping, generalization, and weaning. These techniques are included in other biofeedback protocols and are the basis of biofeedback therapy. ${ }^{11}$ Shaping is a technique for teaching a new motor skill gradually, through successive approximations. Initially, the goal is set at a level the subject can achieve $50 \%-75 \%$ of the time, and as performance improves, the goal is adjusted upward. Generalization involves the use of prescribed home practice program to ensure that skills learned during treatment visits can be applied when needed at home and work. Examples are preventing leakage by "squeezing before you sneeze," becoming "hypervigilant" to notice any rectal sensations and squeezing even if unsure, and "stop, don't run" to the toilet when an urge sensation is felt to allow time for squeezing pelvic floor muscles and relaxing abdominal wall muscles. Weaning refers to eliminating dependence on the equipment and the interventionist's verbal guidance by having the participant practice squeezing without feedback on some squeezes.

The interventionist is trained to assume a coaching role, encouraging and guiding the participant to meet the goals for the biofeedback protocol (Table 2). The goals set for the biofeedback protocol are based on current clinical trial evidence and expert opinion. ${ }^{7-11}$ Visual feedback provided by the computer monitor is supplemented by frequent verbal feedback (Fig. 1). Verbal feedback takes the form of praise for successes, encouragement when the participant's effort approaches but falls just short of the goal, and supportive comments when they fail to reach the goal and seem discouraged. In addition to this verbal feedback, the interventionist should remind the participant how their squeeze and sensory efforts compare to prior visits and how improvements in their squeeze and sensory efforts relate to their goal of eventually achieving continence. The interventionist prescribes a home exercise program and an exercise log based on the session results.

\section{4 | Biofeedback training and certification process}

Prior to participation in the centralized training, trainees were required to review the manual of procedures, complete seven e-learning modules, and review demonstration videos (Table 3). Setup and use of the Medspira MCOMPASS system at their respective institutions was also required.

The in-person certification was a 3-day program which included a "train-the-trainer" day, a training day for trainees, and then a certification day. The "train-the-trainer" day was focused on final confirmation of the protocol among the development team and all evaluators 
TAB LE 3 Certification electronic learning (e-Learning) modules and assessment

\begin{tabular}{|c|c|c|}
\hline e-Learning modules & Content & Completion rate, $\mathrm{N}=24$ \\
\hline Study protocol overview: CAPABLe & $\begin{array}{l}\text { - Protocol background } \\
\text { - Primary and secondary outcomes } \\
\text { - Masking } \\
\text { - Study flow }\end{array}$ & $24(100 \%)$ \\
\hline Anorectal anatomy and physiology overview & $\begin{array}{l}\text { - Pelvic floor anatomy } \\
\text { - Anorectal anatomy } \\
\text { - Defecation process }\end{array}$ & $24(100 \%)$ \\
\hline Biofeedback background principles and protocol overview & $\begin{array}{l}\text { - Biofeedback terminology } \\
\text { - Biofeedback principles } \\
\text { - Protocol goals }\end{array}$ & $24(100 \%)$ \\
\hline Anorectal manometry & $\begin{array}{l}\text { - Catheter setup and proper use } \\
\text { - Equipment setup and use } \\
\text { - Data acquisition from reports } \\
\text { - Troubleshooting }\end{array}$ & $22(92 \%)$ \\
\hline Biofeedback equipment overview & $\begin{array}{l}\text { - Catheter setup and proper use } \\
\text { - Equipment setup and use } \\
\text { - Protocol determination } \\
\text { - Data acquisition from reports } \\
\text { - Troubleshooting }\end{array}$ & $24(100 \%)$ \\
\hline Bowel diary review & $\begin{array}{l}\text { - Education on completion } \\
\text { - Interpretation of findings/symptoms } \\
\text { - Application to protocol }\end{array}$ & $23(96 \%)$ \\
\hline Exercise prescription and record & $\begin{array}{l}\text { - Use of biofeedback data to guide prescription } \\
\text { - Exercise goal attainment } \\
\text { - Instruction on recording exercises }\end{array}$ & $24(100 \%)$ \\
\hline Video demonstration & $\begin{array}{l}\text { - Equipment setup } \\
\text { - Participant interaction } \\
\text { - Participant education } \\
\text { - Anorectal manometry } \\
\text { - Biofeedback protocols }\end{array}$ & $24(100 \%)$ \\
\hline Average completion rate $(n=24)$ & & $98 \%$ \\
\hline
\end{tabular}

and setup of training stations. The second day included education, demonstration, practice, simulation, and feedback with the use of standardized patient volunteers in the Cleveland Clinic Simulation and Advanced Skills Center led by the CAPABLe principal investigator. The trainees were divided into small groups of 3-4 and rotated through five training stations using a valid and reliable skills training format. ${ }^{13-15}$ At each station, each trainee had the opportunity to practice and develop skills with the standardized patients (Table S2). At the completion of all five stations, there was an hour of open practice allowed for the trainees.

The final day of the in-person certification began with a question and answer session. Each trainer was then assigned to a room with live video streaming and recording capabilities, to allow for auditing of the certification process. A standardized patient was provided for each room. One trainer assessed each trainee in a 1:1 (assessor:trainee) ratio using a checklist (Table S2). Assessment checklists were developed using valid and reliable Procedure-Based Assessment (PBA) format. ${ }^{16-19}$ Each item on the list was scored with $\mathrm{N}$ (not observed), D (development required), or S (satisfactory-no prompting or intervention required). A pass rate of $80 \%$ was required for certification. Trainees who were unable to successfully complete the testing were offered re-testing with a different trainer the same day. Trainees who failed evaluation by two different evaluators were required to attend a second in-person training session.

After the in-person training session, the assessors gathered feedback on the training from the interventionists for three educational domains: (i) meeting training objectives, (ii) satisfaction with the quality of education provided, and (iii) confidence in professional ability to complete the protocol. The survey included four response options: "strongly agree," "agree," "disagree," and "strongly disagree." The percentage of survey participants who reported "agreed/strongly agreed" was reported.

\section{5 | Centralized quality monitoring and auditing process}

Quality monitoring of the certified interventionists occurred for the first (baseline) and third visits ( 4 week) from at least two CAPABLe study participants early in the study (first 3 months) and later in the study (after the first year). All visits were recorded with digital audio recording devices. Each site selected two participants for auditing purposes. Auditors included behavioral psychology, physical therapy, nursing, and physician expertise in the treatment of Fl. Encrypted recordings of the intervention session, electronic copies of 
the ARM and biofeedback session summary data generated by the equipment were sent to the auditors. Supporting documents for the audit included the bowel diary, the exercise prescription, the exercise record, the ARM case report form, the equipment summary report, and the interventionist log. A checklist based on the in-person certification checklists was created for the audit (Table S3). The auditors set a minimum pass rate of $80 \%(0 \%-100 \%$ completion of checklist items) for the auditing process. Interventionists who passed the audit received written feedback. Interventionists who did not reach the $80 \%$ pass rate had 1:1 teleconferences set up with the auditor/trainer for remediation.

\section{3 | RESULTS}

The Pelvic Floor Disorders Network (PFDN) consists of a single data coordinating center and eight clinical centers. Centralized training was done at the Cleveland Clinic due to the availability of training facilities. Trainers were picked from the PFDN clinical sites based on personal expertise and either development of or familiarity with the biofeedback protocol. The six trainers included a clinical psychologist, three physicians, an advanced practice registered nurse, and a physical therapist. All had prior experience with pelvic floor therapy for incontinence. All trainers attended the first in-person certification for the first 18 trainees and 3 trainers attended a second certification with 6 trainees in attendance.

Twenty-four interventionists attended the in-person training and certification. Each clinical site had at least two interventionists including 46\% (11/24) advanced practice registered nurses, 50\% (12/24) physical therapists, and $4 \%(1 / 24)$ physician assistants. Interventionists completed a majority of the online training modules (98\%), Table 3. Eight percent $(2 / 24)$ did not pass the initial centralized certification process and one subsequently passed after re-certification. Incorrect equipment usage during the certification limited the passing rate for the two individuals. After the in-person training, interventionists completed an anonymous survey. From those responding to the survey (20/24 or $83 \%$ response rate), $95 \%$ agreed or strongly agreed that training objectives were met, $85 \%$ were satisfied with the quality of the education, and $95 \%$ were confident in their professional ability to complete the protocol.

Audio audits were completed for $88 \%(21 / 24)$ of the certified interventionists with study visits. Two certified interventionists did not see any CAPABLe participants and one did not pass the certification process. Auditors reviewed 84 study visits; $80 \%$ (67/84) had complete audio files, $15 \%(13 / 84)$ had incomplete audio, and 5\% (4/84) were missing audio files. Supporting documents available for the audited visits included the biofeedback summary report (95\%), the ARM case report form (91\%), the bowel diary (82\%), the exercise records/log (86\%), and the interventionist log (91\%). All certified interventionists met or exceeded the audit process for the audited study visits with an average score of $93 \%$.

\section{4 | DISCUSSION}

The CAPABLe protocol is a rigorous, multicenter trial of first-line treatments for $\mathrm{FI}^{12}$ including unique behaviorally based manometric biofeedback clinical protocols addressing each of the major physiologic deficits currently implicated in FI. Specifically, while the strength training component of biofeedback has been fairly well studied, the sensory training to maximize perception of rectal filling and/or tolerance for urge sensations has been more variably described. $^{7,8}$ The ability to separate these aspects of the biofeedback intervention, and to adapt the clinical protocol to the specific deficits of that individual patient by an algorithm, offers the potential for maximizing consistency and reproducibility as well as effectiveness. The centralized training protocol, certification process, and auditing process, as well as the new computer tablet design with on-screen support and prompts, all serve to enhance the internal validity and the reproducibility of the biofeedback interventions in this multicenter design.

The centralized training, certification of interventionists using standardized patients, and auditing of the ARM evaluation and biofeedback training further enhances both the internal and external validity for this intervention. Previous randomized controlled trials of biofeedback for the treatment of FI yielded conflicting results, ${ }^{5}$ and this is sometimes attributed to variability in the training of interventionists. ${ }^{4} \mathrm{~A}$ limitation could be the use of specialized academic medical centers, but generalization of this training protocol is feasible for many types of clinicians caring for patients with $\mathrm{Fl}$ in a variety of clinical settings. We also found limited clinical trial evidence to support specific goals for the three biofeedback protocols for strength and sensitivity training. However, we utilized the best available evidence and expert opinion to develop our comprehensive biofeedback protocol. ${ }^{7-11}$

We realize that using eight separate clinical centers to perform standardized ARM and manometric biofeedback introduces protocol adherence difficulties. Despite geographic diversity, all interventionists received standardized, centralized training in order to minimize variations in protocol performance and measurement. We did not have the ability to give the interventionists live, real-time feedback on their manometry. Instead, we collected a subsample of the data and audited it for outliers using a standardized checklist (Table S3), derived from the items included in interventionist certification. Interventionists were given written feedback when feasible. Another limitation was not using an external auditor to review the manometry and biofeedback tracings for internal consistency. Contracting an external auditor would have provided us with blinded adjudication of our data. However, our internal audits were quite comprehensive and included all components of the visits: audio files, bowel diaries, exercise prescriptions, exercise records, interventionist logs, electronic tracings of the ARM and biofeedback sessions, and the manometry case report forms.

Centralized training including online modules and in-person certification of interventionists of multiple disciplines using standardized patients, and auditing of the biofeedback study visits further enhances 
both the internal and external validity for an evidence-based multicomponent biofeedback protocol.

\section{ACKNOWLEDGMENTS}

Full listing per PFDN guidelines will be submitted upon final acceptance. On behalf of the Pelvic Floor Disorders Network, we would like to thank the interventionists who participated in the certification process and the clinical trial visits. We would also like to thank the standardized patient volunteers and all the women participants in the clinical trial.

\section{FUNDING}

Supported by grants from the Eunice Kennedy Shriver National Institute of Child Health and Human Development (U10 HD054215, U10 HD041261, U10 HD069013, U10 HD054214, U10 HD041267, U10 HD069025, U10 HD069010, U10 HD069006, U01 HD069031) and the National Institutes of Health Office of Research on Women's Health.

\section{DISCLOSURE}

Alayne D. Markland, Ingrid Harm-Ernandes, J. Eric Jelovsek, Jeannine McCormick, Susan Meikle, Diane K. Newman, Gary Sutkin, Amanda Shaffer: No conflict. Uduak U. Andy, Sara Cichowski, Keisher Dyer, Charles Rardin: Research Support - Pelvalon Inc. William E. Whitehead: Research support - Salix Pharmaceuticals; Consultant Ferring Pharmaceuticals.

\section{AUTHOR CONTRIBUTION}

ADM, UUA, KD, IH-E, JEJ, SM, DKN, AS, WEW: concept design, methods, data analysis, and manuscript writing; SC, JMC, CR, GS: concept design, data analysis, and manuscript writing.

\section{REFERENCES}

1. Bharucha AE, Dunivan G, Goode PS, et al. Epidemiology, pathophysiology, and classification of fecal incontinence: state of the science summary for the National Institute of Diabetes and Digestive and Kidney Diseases (NIDDK) Workshop. Am J Gastroenterol. 2015;110:127136.

2. Boreham MK, Richter HE, Kenton KS, et al. Anal incontinence in women presenting for gynecologic care: prevalence, risk factors, and impact upon quality of life. Am J Obstet Gynecol. 2005;192:16371642.

3. Norton NJ. The perspective of the patient. Gastroenterology. 2004;126(1 Suppl 1):S175-S179.
4. Whitehead WE, Rao SS, Lowry A, et al. Treatment of fecal incontinence: state of the science summary for the National Institute of Diabetes and Digestive and Kidney Diseases Workshop. Am J Gastroenterol. 2015;110:138-146.

5. Norton C, Cody JD. Biofeedback and/or sphincter exercises for the treatment of faecal incontinence in adults. Cochrane Database Syst Rev. 2012;7:CD002111.

6. Bharucha AE, Rao SS. An update on anorectal disorders for gastroenterologists. Gastroenterology. 2014;146:37-45 e32.

7. Bols EM, Berghmans BC, Hendriks EJ, et al. A randomized physiotherapy trial in patients with fecal incontinence: design of the PhysioFITstudy. BMC Public Health. 2007;7:355.

8. Bols E, Berghmans B, de Bie R, et al. Rectal balloon training as add-on therapy to pelvic floor muscle training in adults with fecal incontinence: a randomized controlled trial. Neurourol Urodyn. 2012;31:132-138.

9. Bartlett L, Sloots K, Nowak M, Ho YH. Biofeedback for fecal incontinence: a randomized study comparing exercise regimens. Dis Colon Rectum. 2011;54:846-856.

10. Schwandner T, Konig IR, Heimerl T, et al. Triple target treatment (3T) is more effective than biofeedback alone for anal incontinence: the 3T-Al study. Dis Colon Rectum. 2010;53:1007-1016.

11. Heymen S, Scarlett $Y$, Jones K, Ringel $Y$, Drossman D, Whitehead WE. Randomized controlled trial shows biofeedback to be superior to pelvic floor exercises for fecal incontinence. Dis Colon Rectum. 2009;52:1730-1737.

12. Jelovsek JE, Markland AD, Whitehead WE, et al. Pelvic Floor Disorders Network. Controlling anal incontinence in women by performing anal exercises with biofeedback or loperamide (CAPABLe) trial: design and methods. Contemp Clin Trials. 2015;44:164-174.

13. Martin JA, Regehr G, Reznick R, et al. Objective structured assessment of technical skill (OSATS) for surgical residents. Br J Surg. 1997;84:273-278.

14. Faulkner H, Regehr G, Martin J, Reznick R. Validation of an objective structured assessment of technical skill for surgical residents. Acad Med. 1996;71:1363-1365.

15. Reznick R, Regehr G, MacRae H, Martin J, McCulloch W. Testing technical skill via an innovative "bench station" examination. Am J Surg. 1997;173:226-230.

16. Beard JD, Jolly BC, Newble DI, Thomas WE, Donnelly J, Southgate LJ. Assessing the technical skills of surgical trainees. Br J Surg. 2005;92:778-782.

17. James K, Cross K, Lucarotti ME, Fowler AL, Cook TA. Undertaking procedure-based assessment is feasible in clinical practice. Ann $R$ Coll Surg Engl. 2009;91:110-112.

18. Marriott J, Purdie H, Crossley J, Beard JD. Evaluation of procedurebased assessment for assessing trainees' skills in the operating theatre. Br J Surg. 2011;98:450-457.

19. Jelovsek JE, Kow N, Diwadkar GB. Tools for the direct observation and assessment of psychomotor skills in medical trainees: a systematic review. Med Educ. 2013;47:650-673.

\section{SUPPORTING INFORMATION}

Additional Supporting Information may be found online in the supporting information tab for this article. 